\title{
Snap-Through Twinkling Energy Generation Through Frequency Up-Conversion
}

\author{
Smruti R. Panigrahi*a, Brian P. Bernard ${ }^{b}$, Brian F. Feeny ${ }^{a}$, Brian P. Mann ${ }^{b}$, and Alejandro R. Diaz ${ }^{a}$ \\ $a_{428}$ S. Shaw Lane, Michigan State University, East Lansing, MI, 48824, USA \\ ${ }^{b}$ Box 90300, Hudson Hall, Duke University, Durham, NC, 27708, USA
}

\begin{abstract}
A novel experimental energy harvester is investigated for its energy harvesting capability by frequency up-conversion using snap-through structures. In particular, a single-degree-of-freedom (SDOF) experimental energy harvester model is built using a snap-through nonlinear element. The snap-through dynamics is facilitated by the experimental setup of a twinkling energy generator (TEG) consisting of linear springs and attracting cylindrical bar magnets. A cylindrical coil of enamel-coated magnet wire is used as the energy generator. The governing equations are formulated mathematically and solved numerically for a direct comparison with the experimental results. The experimental TEG and the numerical simulation results show 25 -fold frequency up-conversion and the power harvesting capacity of the SDOF TEG.
\end{abstract}

Keywords: vibration energy harvesting, renewable energy, low-frequency vibration, frequency up-conversion, negative stiffness, snap-through oscillators

\section{INTRODUCTION}

There is an abundance of energy available in the low frequency range, but the conversion of this mechanical energy into usable form for micro-scale devices is a primary concern. For example, consider harvesting energy from a micro-scale beam. A primary concern is the beam natural frequency would typically be in the tens of $\mathrm{kHz}$, but the bulk of the available energy is in the low tens of $\mathrm{Hz}$, i.e. maybe below $25 \mathrm{~Hz}$ in many applications. Twinkling phenomenon could potentially be used to up-convert low-frequency excitations into a usable frequency range. Twinkling occurs when a nonlinear spring-mass snapthrough chain is loaded slowly and the masses snap-through, converting the low-frequency input to high-frequency oscillations. In this paper, a demonstration of an energy harvester that exploits the up conversion in frequency associated with the twinkling phenomenon is presented.

Vibration-based energy harvesting has recently gained lot of attention. Nonlinear spring-mass systems, such as snap-through structures, have the potential to harvest energy from a variety of low-frequency sources. To be able to harvest such energy, it is critical to understand the underlying dynamics of the coupled snap-through oscillators. Several authors have studied the dynamics of various snap-through negative-stiffness and bistable systems [1]-[8]. Vibration-based energy harvesting from linear systems [9], [10] has been optimized experimentally [11]-[13] by tuning the forcing frequency to the natural frequency of the oscillator. Piezoelectric materials are used for successful experimental energy harvesting from vibrating sources [9], [10], [14]-[17] and fluctuating pressure load [18], [19].

Nonlinearity has been studied by various authors for energy management. For example, essential nonlinearity has been used as a nonlinear energy sink (NES) for energy harvesting [20]-[23], nonlinear energy pumping [24]-[27], and nonlinear targeted energy transfer (TET) [28]-[33]. Various authors have conducted experimental energy harvesting for low-frequency ambient excitations [15], [16], [34]-[36], nonlinear oscillations of magnetic levitations [37], and snap-through oscillators [16], [38]-[46].

The vibration-based energy harvesting in this article is based on the nature-inspired dynamics of the hairs in bull-frog's ears [47], [48] that exhibit bistable properties [49], [50]. Recently there has been growing interest in designing materials that can exhibit multiple stable equilibrium states and help channel the flow of energy in a desirable direction and/or for a desirable frequency. One way to achieve this is by using bistable elements in a mass-spring chain. There are two stable equilibrium states for every bistable element. Though the stability of a single-degree-of-freedom (SDOF) bistable structure seems simple, when more bistable elements are added, it forms a complex dynamical system and exhibits multiple equilibria [51], [52]. These bistable elements, when combined, enable high-frequency oscillations. This property of a bistable chain can be exploited in order to convert low-frequency vibrations into high-frequency oscillations, which in turn can be used for energy harvesting and energy transfer. This paper takes a step toward demonstrating such a capability by exploiting frequency up-conversion of twinkling in a SDOF pulled snap-through mass-spring to harvest energy.

This work explores snap-through twinkling and energy harvesting by using magnets and coils. The main idea of this research is to increase the frequency in order to take advantage of a magnet-coil harvester system that performs best for fast oscillations of the magnet inside the coil. The frequency up-conversion is done using the snap-through phenomenon. In this research

corresponding author email: smrutiranjan@gmail.com.

(C) 2017. This manuscript version is made available under the Elsevier user license

http://www.elsevier.com/open-access/userlicense/1.0/ 
frequency up-conversion is done by using attracting magnets and linear springs in a novel fashion to form a snap-through system. It is demonstrated that by using the snap-through phenomenon one can increase the response frequency more than 25-fold for a low frequency vibration excitation input.

The work is organized as follows. In Section II, the experimental setup and the corresponding physical parameters are presented. Keeping the experimental setup in focus, we describe the system mathematically in Section III. In this section the forces due to the linear springs, the nonlinear magnetic attraction forces between cylindrical magnets, and the idle equilibrium state force balance are discussed. The numerical simulation results are presented in Section IV. In Section V, the experimental results for harvested power are presented in comparison with the numerical simulations of the analytical models to confirm the power harvesting capacity, and the frequency up-conversion as well as the broadening of the frequency spectrum. Finally, the results obtained in this paper are summarized in Section VI.

\section{EXPERIMENTAL SETUP OF THE TWINKLing ENERgy GENERATOR}

In this section the details of the experimental setup and the experimental parameter values are described. The experimental parameter values are later used in the mathematical model for obtaining the numerical simulation results. A SDOF experimental snap-through twinkler is built for power harvesting from low-frequency input oscillation. Snap-through is achieved with attracting magnets which are "locked" in contact until the force of a pulled spring overcomes the attraction force, at which point the magnets release and the mass can oscillate about a non-contact equilibrium.

The experimental setup consisted of an air track for low mechanical damping, a horizontal shaker to induce a low-frequency vibration input, a triangular shaped cart as the moving mass with 3D-printed mounts for the magnets, and a combination of linear springs and cylindrical bar magnets to induce snap-through in the system as well as to facilitate power generation in a coil made of enamel-coated magnet wire, as shown in Figure 1. The mass consisted of the moving cart, a magnet mount, and a magnet. The mass was constructed in such a fashion that the magnets were fixed to the magnet mounts using nuts and bolts. A stationary magnet was attached through a magnet mount to a fixed base (the fixed cart in Figure 1). Beads were attached to the magnets to provide a spacer of distance $d_{m}$ that reduces the attractive force between the magnets when they are "locked" in the idle state. The beads also avert direct impact between the magnets and prevent impact damage. The harvester coil was mounted outside the air track such that when the mass-mounted magnet cylindrical bar magnet was passed through the center of the coil, power was generated. Figure 1 (a) shows the twinkling energy generator (TEG) in its idle equilibrium state, for which the magnets are in contact through the spacer beads, and (b) shows the TEG in its dynamic state when the magnets are detached and the magnet oscillates inside the harvester coil, which was located a distance $x_{0_{\text {coil }}}$ from the idle position of the oscillating magnet. Springs with different stiffnesses were used in various setups of the SDOF TEG experiment. Generator models with both unsymmetric springs with different stiffnesses and symmetric springs with almost identical stiffnesses were investigated.

An electrical circuit was set up in order to capture the voltage output generated in the harvester coil when the oscillating magnet passes through the inducting coil. The horizontal shaker initiated through a MATLAB script was used to induce external excitation and the generator coil was connected to the circuit board that fed the output analog signal (voltage) into a data acquisition board (DAB). This DAB converted the analog output into a digital signal. The output digital signal was the voltage output generated in the generator coil. The mechanical, magnetic, and electrical circuit parameter values are tabulated in Table I.

\section{Mathematical Model of the Twinkling Energy Generator}

The mathematical model of the nonlinear twinkling energy generator is formulated to understand the dynamics and compare with the experiments. A schematic diagram of the experiment (Figure 2) serves to facilitate the mathematical model. The equations of motion of the TEG consists of both forces due to the magnets as well as the linear springs, which are derived in this section.

TABLE I: Spring, Mass, Magnet, and Electrical Circuit Parameters Used in the TEG

\begin{tabular}{lclclc}
\hline Mechanical Parameters & Value & Magnet Parameters & Value & Electrical Circuit Parameters & Value \\
\hline \hline$m(\mathrm{~kg})$ & 0.342 & $w_{m}(\mathrm{~kg})$ & 0.009 & $R_{L}(\Omega)$ & 2000 \\
$c(\mathrm{Ns} / \mathrm{m})$ & 0.01 & $l_{m}(\mathrm{~m})$ & 0.0127 & $R_{i}(\Omega)$ & 200 \\
$c_{e}(\mathrm{Ns} / \mathrm{m})$ & 0.01 & $d_{o}(\mathrm{~m})$ & 0.0127 & $n$ & 2000 \\
$k(\mathrm{~N} / \mathrm{m})$ & 44.10 & $d_{i}(\mathrm{~m})$ & 0.00635 & $r_{c i}(\mathrm{~m})$ & 0.016 \\
$k_{e}(\mathrm{~N} / \mathrm{m})$ & 45.52 & $d_{m}(\mathrm{~m})$ & 0.0145 & $l_{c}(\mathrm{~m})$ & 0.022 \\
$\Delta x_{0}(\mathrm{~m})$ & 0.088 & $x_{0_{c o i l}}(\mathrm{~m})$ & 0.025 & $L(\mathrm{H})$ & 5 \\
$A_{0}(\mathrm{~m})$ & 0.1524 & $M(\mathrm{~A} / \mathrm{m})$ & $1.05 \times 10^{6}$ & $\gamma_{\text {model } 1}(\mathrm{As} / \mathrm{m})$ & 0.64 \\
$f_{0}(\mathrm{~Hz})$ & 0.1 & $\mu\left(\mathrm{H} / \mathrm{m}\right.$ or N/A $\left.{ }^{2}\right)$ & 1.05 & $\gamma_{\text {model } 2}($ As $/ \mathrm{m})$ & 1.1 \\
\hline
\end{tabular}




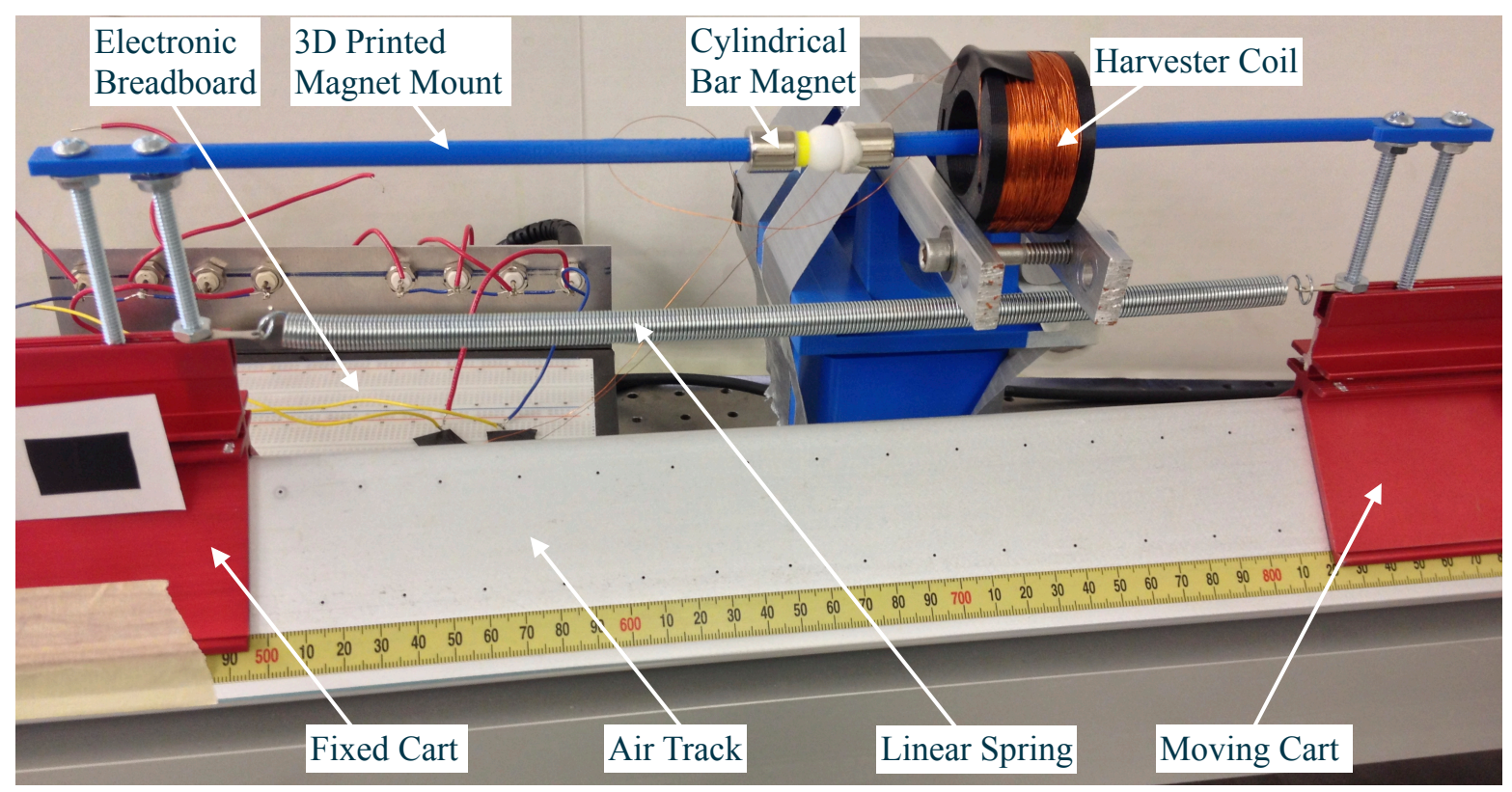

(a)

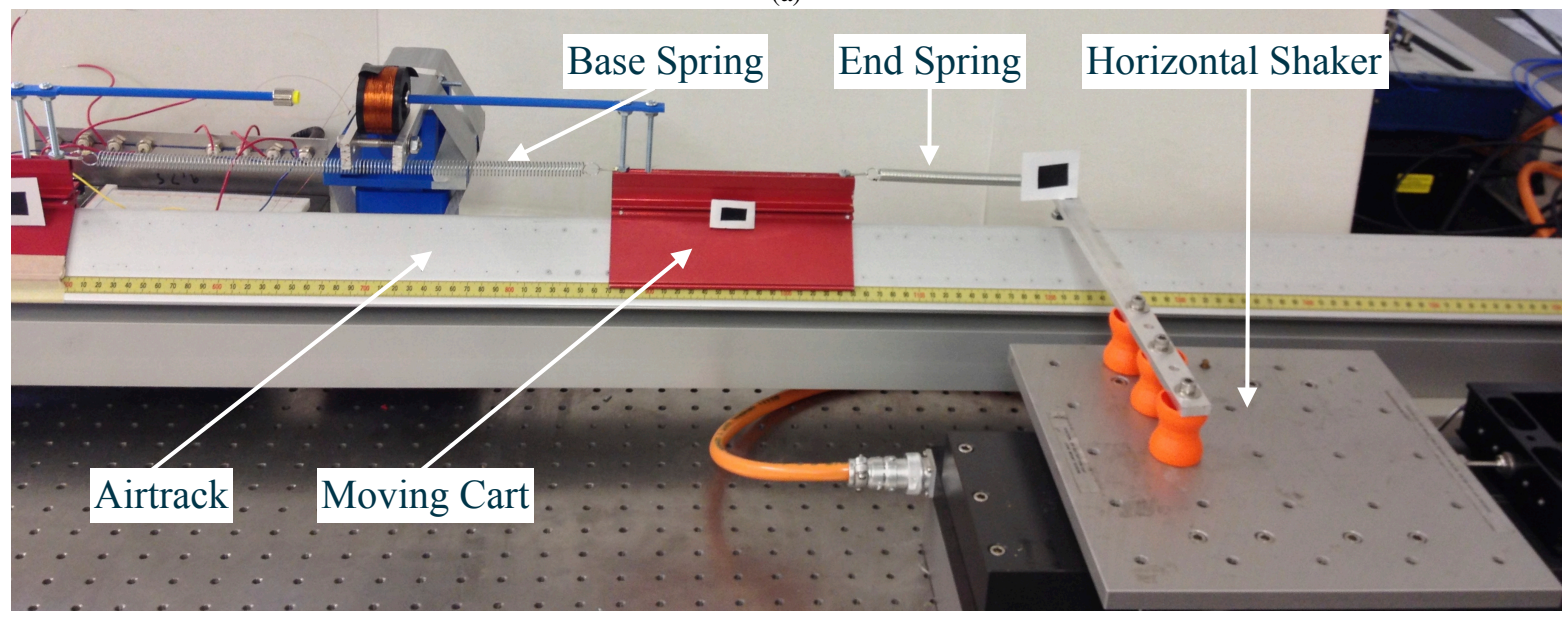

(b)

Fig. 1: Shown here is the TEG experimental setup on the air track. (a) The idle equilibrium state of the TEG consisting of the primary mass (the cart), the magnets, the magnet mounts, and a linear spring, when the magnets are connected through the spacer beads. (b) The TEG in its dynamic state when the magnets are detached and the magnet oscillates inside the generator coil. The right spring in (b) is pulled by a horizontal shaker with a stroke length of 6 inches.

\section{A. Force Due to Linear Springs}

The spring forces due to the linear springs in the SDOF TEG as shown in Figure 2 (a), are

$$
\begin{aligned}
& F_{s}=k\left(\Delta x_{0}+x(t)\right) \\
& F_{s_{e}}=k_{e}(y(t)-x(t))
\end{aligned}
$$

where $F_{s}$ and $F_{s_{e}}$ are the linear spring forces of the spring attached to the fixed base and the spring force of the end spring (connected to the shaker) respectively, $k_{e}$ is the stiffness of the end spring, $k$ is the stiffnesses of the spring connected to the fixed base, $y(t)$ is the low-frequency pull on the end spring, $x(t)$ is the displacement of the mass, and $\Delta x_{0}$ is the initial stretch of the spring connected to the fixed base in the idle equilibrium configuration $(x(t)=0)$.

The initial stretch, $\Delta x_{0}$, in the idle equilibrium configuration is computed from the following relations:

$$
\Delta x_{0}=d_{c}-l_{s}
$$

where $d_{c}=0.326 \mathrm{~m}$ is the distance between the cart and the fixed base, and $l_{s}=0.238 \mathrm{~m}$ is the undeformed length of the spring attached to the fixed base.

In the $x=0$ configuration, the magnets are connected as in Figure 1 (a) and the linear spring is stretched by $\Delta x_{0}$. 


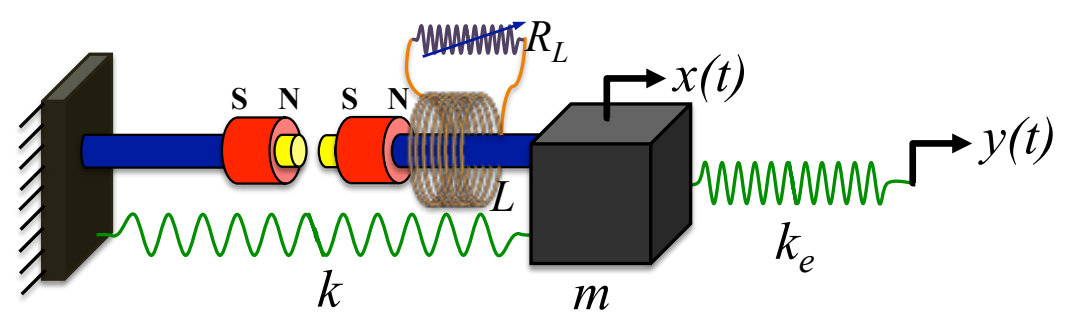

(a)

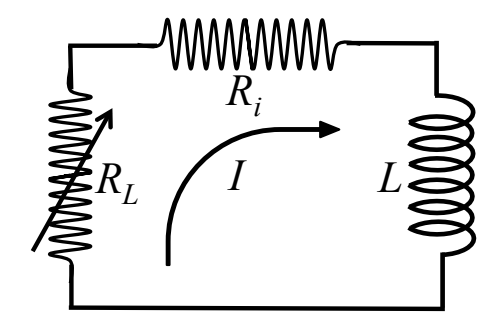

(b)

Fig. 2: (a) The SDOF twinkling oscillator schematic consisting of one cart (black), magnet mounts (blue), magnets (red), beads (yellow) of effective length $d_{m} / 2$ to maintain a separation of $d_{m}$ between the magnet centers in the steady state, and linear springs. Mass $m$ is the sum of the weights of the cart, the magnet mount, magnet, the separating-beads, and the springs. $k$ is the stiffness of the spring connected to the left base, and $k_{e}$ is the stiffness of the end spring. On the magnets, $N$ and $S$ represent the north and south poles of the cylindrical magnets respectively. When the magnet passes through the coil with inductance $R_{L}$ it generates current in the coil and powers the resistive load $R_{L}$. Here $x(t)$ is the dynamic displacement of the cart and $y(t)$ is the horizontal excitation input. When $x=0$, the magnet beads are in contact and the spring $k$ is stretched by $\Delta x_{0}$. (b) The electrical circuit in the energy generator consisting of an inductor $(L)$, a variable resistor with resistance $\left(R_{L}\right)$, and internal resistance of the circuit $\left(R_{i}\right)$.

\section{B. Force Due to Magnets: Model I}

In this model, the magnetic force is assumed to follow a point dipole approximation. This approximation holds true when the lengths, $l$, of the cylindrical bar magnets are much smaller compared to the separation, $x$, between them $(l<<x)$ which is often referred to as Gilbert's model, named after the sixteenth century British physicist and astronomer William Gilbert [53]. Keeping this assumption in mind, the magnetic forces between two point-dipole magnets are derived below.

The magnetic field strength, $\mathbf{B}$, at location $\mathbf{r}_{f}$ due to a point-dipole magnet (representing the oscillating magnet) located at $\mathbf{r}_{o}$ is

$$
\mathbf{B}=-\frac{\mu_{0}}{4 \pi} \nabla\left(\frac{\mathbf{m}_{o} \cdot \mathbf{r}_{f / o}}{\left|\mathbf{r}_{f / o}\right|^{3}}\right)=-\frac{\mu_{0}}{4 \pi}\left(\frac{\mathbf{m}_{o}}{\left|\mathbf{r}_{f / o}\right|^{3}}-\frac{3 \mathbf{r}_{f / o}\left(\mathbf{m}_{o} \cdot \mathbf{r}_{f / o}\right)}{\left|\mathbf{r}_{f / o}\right|^{5}}\right)
$$

where, $\mu_{0}=4 \pi \times 10^{-7} \mathrm{H} / \mathrm{m}$ is the permeability of free space, $\mathbf{m}_{o}$ is the magnetic moment of the oscillating magnet at location $\mathbf{r}_{o}, \nabla$ is the vector gradient, $\mathbf{r}_{f / o}=\mathbf{r}_{f}-\mathbf{r}_{o}$, is the vector to a point of interest (to the fixed magnet in our case) from the oscillating magnet, and $\left|\mathbf{r}_{f / o}\right|$ is the distance between the oscillating and the fixed magnet.

In reference to the arrangements in Figure 2 (a) $\mathbf{m}_{o}=m_{o} \mathbf{i}, \mathbf{r}_{f / o}=-\left(d_{m}+x\right) \mathbf{i},\left|\mathbf{r}_{f / o}\right|=\left(d_{m}+x\right)$, and $\mathbf{m}_{o} \cdot \mathbf{r}_{f / o}=$ $\left(d_{m}+x\right) m_{o}$, where $\mathbf{i}$ is the unit vector in the axis of displacement and $d_{m}$ is the distance between the magnets due to attached beads and putty to the magnet mounts to reduce the attracting force on the magnets and maintain a separation between the magnets in the stable configuration. Also the magnitude of the magnetic moment of a magnet is given as $m_{o}=M_{o} V_{o}$, where $M_{o}$ is the magnetization and $V_{o}$ is the volume of the magnet. Hence the magnetic strength is

$$
\mathbf{B}=-\frac{\mu_{0}}{4 \pi}\left[\frac{m_{o} \mathbf{i}}{\left(d_{m}+x\right)^{3}}-\frac{3\left(\left(d_{m}+x\right) \mathbf{i}\right) m_{o}\left(d_{m}+x\right)}{\left(d_{m}+x\right)^{5}}\right]=\frac{\mu_{0} m_{o}}{2 \pi\left(d_{m}+x\right)^{3}} \mathbf{i}
$$

The potential energy of the fixed magnets at $\mathbf{r}_{f}$ with magnetic moment $\mathbf{m}_{f}=m_{f} \mathbf{i}$, in the field generated by the magnet at $\mathbf{r}_{o}$ is

$$
U_{m}=\mathbf{m}_{f} \cdot \mathbf{B}=\frac{\mu_{0} m_{o} m_{f}}{2 \pi\left(d_{m}+x\right)^{3}}
$$

The total magnetic force in the longitudinal direction (along the oscillating magnet) is then computed as

$$
\hat{F}_{m}(x)=\nabla U_{m}=\frac{\partial U_{m}}{\partial x}=-\frac{3 \mu_{0} m_{o} m_{f}}{2 \pi\left(d_{m}+x\right)^{4}}
$$

\section{Force Due to Magnets: Model II}

In deriving the magnetic force in the model I, a Gilbert's point dipole approximation is used for the force between the two attracting cylindrical magnets. However, the forces between two cylindrical permanent magnets derived mathematically in [54] show excellent convergence to the forcing obtained through experiments for cylindrical magnets of length $l_{m}$ in the order of $0.01 \mathrm{~m}$ while separated by a distance of $d_{m}$ in the order of $0.01 \mathrm{~m}$. This promising theoretical derivation [54] of the magnetic forces between two cylindrical bar magnets is used in this TEG model. 
The attracting force between the cylindrical magnets is derived from the gradient of the magneto-static interaction energy $E_{m}$. Hence, for two cylindrical magnets aligned along the $x$-axis, the force is derived as [55]

$$
F_{m}=-\nabla E_{m}=-\frac{\partial E_{m}}{\partial x}=2 \pi \mu_{0} M^{2} R_{m}^{3} \frac{\partial J_{d}}{\partial x}
$$

where $\mu_{0}$ is the permeability of vacuum, $M$ is the magnetization of the cylindrical magnets, $R_{m}$ is the radius of the magnets, and $J_{d}$ is the dipolar coupling integral [56]. The dipolar coupling integral $J_{d}$ for two cylindrical magnets with parallel axes with lateral distance $r$ can be found as

$$
J_{d}\left(\tau_{1}, \tau_{2}, x\right)=2 \int_{0}^{\infty} J_{0}\left(\frac{r \alpha}{R_{m}}\right) \frac{J_{1}^{2}(\alpha)}{\alpha^{2}} \sinh \left(\alpha \tau_{1}\right) \sinh \left(\alpha \tau_{2}\right) e^{-\alpha \eta} d \alpha
$$

where $\alpha$ is an independent integration variable, $\tau_{i}=l_{m_{i}} /\left(2 R_{m}\right), i=1,2$, are the aspect ratios of the two cylindrical magnets, $\eta=\frac{x}{R_{m}}+\tau_{1}+\tau_{2}=\frac{2 x+l_{m_{1}}+l_{m_{2}}}{2 R_{m}}$ is the nondimesional distance between the center of the magnets when separated by a distance $x, J_{0}(\alpha)$ is the modified Bessel function of order zero, and $J_{1}(\alpha)$ is the modified Bessel function of the first kind.

When the magnets are far from each other (large $x$ ), an approximation of the magnetic force is obtain by expanding the Bessel functions around $q=0$ [57] and written as

$$
F_{m x}(r, x) \approx-\frac{1}{4} \pi \mu_{0} M^{2} R_{m}^{4} \sum_{i=0}^{1} \sum_{j=0}^{1} \frac{(-1)^{i+j}}{x_{i j}^{2}}\left[1-\frac{3 r^{2}}{2 x_{i j}^{2}}\right]
$$

where $x_{i j}=\left(x+i l_{m_{1}}+j l_{m_{2}}\right)$.

In the twinkling energy generator the magnets are identical with the magnetization vectors aligned on their common axes, i.e. $r=0, \tau_{1}=\tau_{2}=\tau$, and $l_{m_{1}}=l_{m_{1}}=l_{m}$, resulting in the force between the fixed and the oscillating magnet as

$$
F_{m x}(x) \approx-\frac{1}{4} \pi \mu_{0} M^{2} R_{m}^{4}\left[\frac{1}{x^{2}}+\frac{1}{\left(x+2 l_{m}\right)^{2}}-\frac{2}{\left(x+l_{m}\right)^{2}}\right]
$$

Experimental investigations [54] have shown the above magnetic force to be an excellent approximation when the axial separation between the magnets, $x$, is on the order of the length of the magnets, $l_{m}$.

Since the magnets used in the TEG are cylindrical ring magnets, the magnetic force is modified such that the force is equal to the magnetic force due to interaction between solid cylindrical magnets with diameters $d_{o}$ less the magnetic force due to interaction of solid cylindrical magnets with diameters $d_{i}$. Using this modification to the above magnetic force for the TEG with hollow cylindrical magnets separated by a distance $d_{m}$, the resulting magnetic force can be written as

$$
F_{m}(x)=-\frac{\pi \mu_{0} M^{2}\left(d_{o}^{4}-d_{i}^{4}\right)}{64}\left[\frac{1}{\left(x+d_{m}\right)^{2}}+\frac{1}{\left(x+d_{m}+2 l_{m}\right)^{2}}-\frac{2}{\left(x+d_{m}+l_{m}\right)^{2}}\right]
$$

\section{Force Balance in an Idle Steady State}

At idle steady-state conditions, when the magnets are connected, the total magnetic energy and the spring (connected to the left base or the left cart) potential are non-zero. Hence it would take a certain amount of work to detach the magnets. This work is done by the stretching of the end spring. To mimic the experimental results, the mathematical model needs to balance the energy stored in the magnets and springs with the work done by the pull of the end spring. The total displacement of the end spring needed for the magnet to detach is denoted by $\Delta x_{e}$ and is given by

$$
\Delta x_{e}=\frac{1}{k_{e}}\left[F_{m_{0}}+F_{s_{0}}\right]
$$

where $k_{e}$ is the stiffness of the end spring, $F_{m_{0}}$ is the magnetic force and $F_{s_{0}}$ is the linear base-spring force at the instant when the magnets detach.

The time it would take to detach the springs can be found by using the low-frequency input displacement. In our experiment the pull of the end spring is approximated as

$$
y(t)=\frac{A_{0}}{2}\left(1-\cos \left(2 \pi f_{0} t\right)\right)
$$

where $A_{0}=6$ inches is the stroke length of the horizontal shaker, and $f_{0}=0.1 \mathrm{~Hz}$ is the frequency of the input oscillation by the shaker as shown in Figure 3.

The end spring deformation is $y(t)-x(t)$. So when the magnets are connected $(x=0)$, they release when $y(t)=\Delta x_{e}$. Hence the time taken by the horizontal shaker to detach the magnets from its idle equilibrium state is

$$
\Delta t_{0_{\text {idle }}}=\frac{1}{2 \pi f_{0}} \cos ^{-1}\left(1-\frac{2 \Delta x_{e}}{A_{0}}\right)
$$




\section{E. Governing Equations of Motion of the TEG}

The force between the fixed and oscillating magnets of a twinkler with attracting magnets is first derived using the dipole moments generated by the magnets. Combined with linear springs, the interaction between the magnets form a bistable system. The complete model is then formulated using the electrical circuit consisting of an inducting coil, a transducer, and a resistive load.

The generator coil induces an electrical damping in the SDOF twinkler. The generator is manipulated by the magnets and the springs to scavenge energy induced by the low-frequency pull to the end spring.

The governing equations of motion of the SDOF twinkler shown in the Figure 2 (a), directly powering a resistive load, are

$$
\begin{aligned}
& m \ddot{x}+c \dot{x}+c_{e}(\dot{y}-\dot{x})+F_{s}-F_{s_{e}}+F_{m}-\gamma I=0 \\
& L \dot{I}+\left(R_{L}+R_{i}\right) I+\gamma \dot{x}=0
\end{aligned}
$$

where $F_{m}$ is a nonlinear function of $x$ given in equation (11), $m$ is the combined mass of the magnet and the mount, $c$ and $c_{e}$ are the mechanical dissipation constants, $y$ is the low-frequency pull, $I$ is the electrical current, $R_{L}$ is the resistance of the resistive load, $R_{i}$ is the internal resistance of the electrical circuit, $L \approx \mu_{0} n^{2} \pi r_{c i}^{2} / l_{c}$ is the inductance of the harvester coil where $n, r_{c i}$ and $l_{c}$ are the number of windings in the coil, inner radius of the coil and the length of the coil respectively, and $\gamma$ is a transducer constant.

The magnetic force $F_{m}$ is replaced by $\hat{F}_{m}(x(t))$ from equation (6) when we approximate the magnetic force using model I, and is replaced by $F_{m}(x(t))$ from equation (11) when we use the magnetic force derived in model II. Similarly, the value of the transducer constant $\gamma$ is $\gamma_{\text {model } 1}$ and $\gamma_{\text {model } 2}$ for the magnetic force models I and II respectively.

\section{Numerical Simulation of the Twinkling EnERgy Generator}

The first mathematical model of the magnetic force in the twinkling energy generator in Section III-B is derived with an assumption that the magnetic force follows a point dipole approximation which is true when the length of the cylindrical magnet $\left(l_{m}\right)$ is much smaller compared to the separation between the magnets $\left(d_{m}\right)$. In an idle steady equilibrium state the distance between the two magnets is $d_{m}=0.0145 \mathrm{~m}$ and the length of the magnets is $l_{m}=0.0127 \mathrm{~m}$. Though $l_{m}<d_{m}$, it is clear that the condition $l_{m}<<d_{m}$ is not satisfied. Hence, a deviation of the numerical solutions of the analytical model from the experimental results should be expected.

The magnetic force in the TEG in Section III-C is derived using a more accurate representation of the magnetic interaction forces between two attracting cylindrical magnets. This model is an excellent model to mimic the experiment since both the lengths and the radii of the magnets are in the order of 0.01 meters and the minimum distance $d_{m}$ between the magnet centers is on the order of the length $l_{m}$ of the magnets. Using the magnetic force, from equation (11), between the fixed and oscillating magnets of the twinkler and the spring forces from equation (1), the governing equations of motion of the SDOF nonlinear energy generator are represented in equation (15).

The solutions obtained by applying numerical simulation tools in MATLAB, based on the ode 45 solver, to the analytical models developed in the previous section are presented here. The measured parameter values from the experiment are used to numerically solve the nonlinear equations.

A low-frequency input is applied by pulling the end point of the end spring from the zero position, $y=0$, to a maximum position, and then back to zero, by means of the harmonic function of equation (13). One cycle of input was simulated. With the application of this periodic low-frequency input, the magnets snap and detach after certain time, $\Delta t_{0_{i d l e}}$, evaluated using equation (14), when the spring force in the end spring is just enough to overcome the total force due to the magnets and the left spring. The mass then oscillates and energy is harvested when the oscillating magnet is in the coil. As the input returns toward the zero position, at some time $t_{f}$ the oscillating magnet eventually reattaches with the fixed magnet, and they remain stuck through the end of the cycle.

During the oscillatory phase, the magnets do not pass through the coil until $x(t)$ goes beyond $x_{0_{\text {coil }}}$, where $x_{0_{\text {coil }}}$ is the distance between the magnet at the idle equilibrium state and the left end of the coil spool as shown in Figure 2 (a). The value of $x_{0_{\text {coil }}}$ is given in Table I. Also the current is generated only when the magnet passes inside the coil. No current is generated once the moving magnet passes beyond the length of the coil. To mimic this localized current generation of the magnets in the numerical simulation, the velocity term in the generator equation and the current term in the displacement equations in the above governing equations of motion are multiplied with a combination of Heaviside functions, [heaviside $\left(x-x_{0_{\text {coil }}}\right)$ - heaviside $\left.\left(x-x_{0_{\text {coil }}}-l_{c}\right)\right]$, where $l_{c}$ is the length of the coil.

Since the Heaviside function has a sharp transition between 0 and 1 , in some cases it is better to smoothen the function with stiff hyperbolic tangent functions. As such $\frac{1}{2}\left[\tanh \alpha\left(x-x_{0_{\text {coil }}}\right)-\tanh \alpha\left(x-x_{0_{\text {coil }}}-l_{c}\right)\right]$ can be used to approximate [heaviside $\left(x-x_{0_{\text {coil }}}\right)$-heaviside $\left(x-x_{0_{\text {coil }}}-l_{c}\right)$ ], where $\alpha$ is a very large number and is of $O(100) \mathrm{rad} / \mathrm{inch}$ in our case. For the numerical simulation, the measured and assumed experimental parameter values given in Table I are used.

Since the experimental value for the transducer constant, $\gamma$, was not measured, we have used two different values of $\gamma$ for the numerical simulations corresponding to the two models. For magnet models I and II the transducer constants used are $\gamma_{\text {model } 1}=0.64 \mathrm{As} / \mathrm{m}$ and $\gamma_{\text {model } 2}=1.1 \mathrm{As} / \mathrm{m}$ respectively 


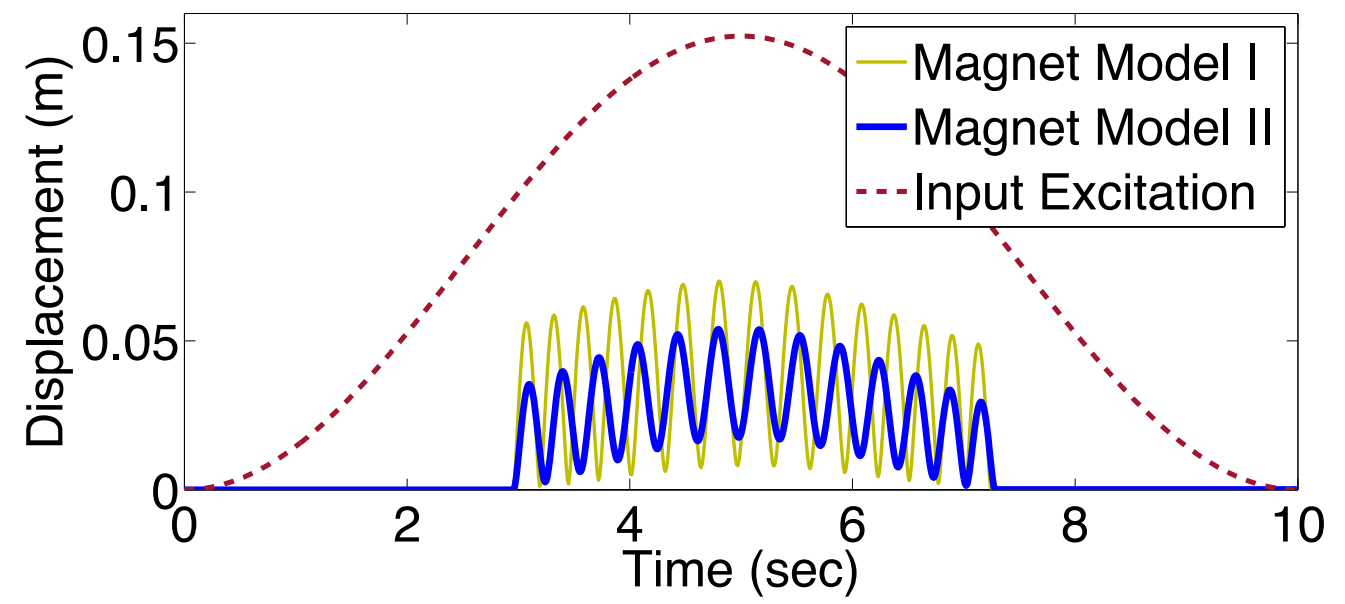

Fig. 3: The numerical displacement output, $x(t)$, of the mathematical model of the SDOF twinkler, for one cycle of $0.1 \mathrm{~Hz}$ input excitation.

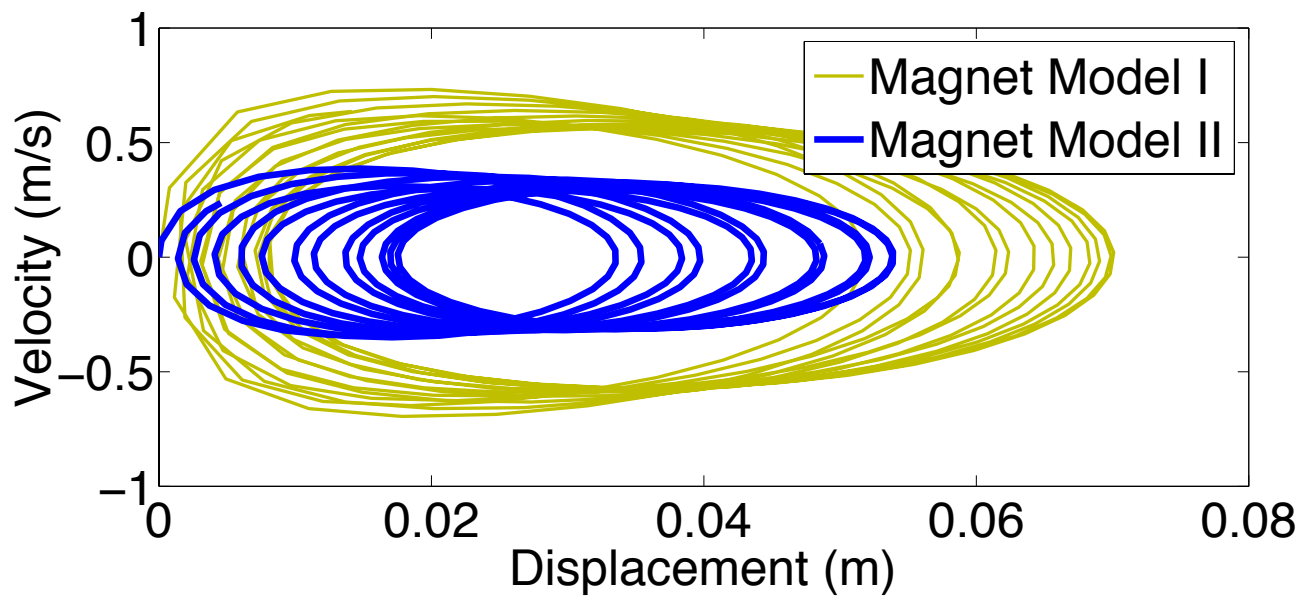

Fig. 4: Phase portrait of the numerical SDOF TEG for one cycle of $0.1 \mathrm{~Hz}$ input excitation

\section{A. State Space Representation of the SDOF TEG}

By changing the end spring and the distance between magnets, various experimentations were performed for the comparison of the power output. To keep the analytical and numerical simulations simple, one such experimental case and the associated governing equations of motion of the SDOF TEG are studied.

We introduce state variables $u=x, v=\dot{x}$, along with the state $I$. For $t<\Delta t_{0_{\text {idle }}}$, the system is in the idle position, and thus $u=0, v=0$, and $I=0$. For $\Delta t_{0_{i d l e}} \leq t \leq t_{f}$, the system is in the oscillatory phase governed by equation (15). The state-space representation of these equations is used in the numerical simulation and is written as

$$
\begin{aligned}
& \dot{u}=v \\
& \dot{v}=-\frac{1}{m}\left[k \Delta x_{0}+\left(c+c_{e}\right) v+\left(k+k_{e}\right) u+F_{m}(u)-\gamma I_{\text {delayed }}-c_{e} \dot{y}(t)-k_{e} y(t)\right] \\
& \dot{I}=-\frac{1}{L}\left[\left(R_{L}+R_{i}\right) I+\gamma v_{\text {delayed }}\right]
\end{aligned}
$$

for $\Delta t_{0_{\text {idle }}} \leq t \leq t_{f}$, where

$$
\begin{aligned}
& I_{\text {delayed }}=\frac{1}{2}\left[\tanh \alpha\left(u-x_{0_{\text {coil }}}\right)-\tanh \alpha\left(u-x_{0_{\text {coil }}}-l_{c}\right)\right] I \\
& v_{\text {delayed }}=\frac{1}{2}\left[\tanh \alpha\left(u-x_{0_{\text {coil }}}\right)-\tanh \alpha\left(u-x_{0_{\text {coil }}}-l_{c}\right)\right] v
\end{aligned}
$$

with input oscillation $y(t)=\frac{A_{0}}{2}\left(1-\cos \left(2 \pi f_{0} t\right)\right)$ and $\dot{y}(t)=\pi A_{0} f_{0} \sin \left(2 \pi f_{0} t\right) . A_{0}$ is the stoke-length of the horizontal shaker, and $f_{0}$ is the frequency of the input oscillation. For the numerical simulation with the chosen parameters, it turns out that the magnets snap back together at $t_{f}=T-0.933 \Delta t_{0_{i d l e}}$, where $T=10$ seconds is the period of input oscillation.

When $t>t_{f}$, and the magnets are attached, we have $u=0, v=0$, and $I=0$. The value of $I=0$ is an approximation, as 
the current would behave as a decaying exponential once the oscillating magnet leaves the coil. With our simulated parameter values, the time constant is very short compared to the excitation period, and thus $I \approx 0$ is valid and practical.

With the $0.1 \mathrm{~Hz}$ input, the simulation results using the magnetic interaction derived in Section III-C are presented for the dynamic displacement of the magnet in Figure 3, the phase portrait in Figure 4, the frequency characteristics of the displacement output in Figure 5, the voltage generated in the harvester coil in Figure 6, the frequency characteristics of the voltage output in Figure 7, and the total harvestable energy in Figure 8. The numerical displacement and voltage results (Figures 5 and 7) clearly show the up-conversion of the input frequency by a factor of about 25-30.

\section{B. Energy and Power Derivation}

The total energy (in Joules), near the linear non-saturated regions of the magnetic flux linkage and current relationship, stored by an inductor is equal to the amount of work required to establish the current through the inductor and is given by

$$
E_{\text {stored }}=\frac{1}{2} L I^{2}
$$

where $L$ is the inductance of the inductor coil and $I$ is the current through the inductor.

In general the energy stored in an inductor that has initial current during a specific time interval $t_{1}$ and $t_{2}$ is

$$
E=\frac{1}{2} L I\left(t_{2}\right)^{2}-\frac{1}{2} L I\left(t_{1}\right)^{2}
$$

Since the electrical circuit in the TEG is such that it directly powers a resistive load, the instantaneous power delivered to the electrical load is given by

$$
P(t)=I(t) \cdot V(t)=I(t)^{2} R_{L}=\frac{V(t)^{2}}{R_{L}}
$$

The average power $P_{a v g}$ over a period of time $T$ is given as

$$
P_{\text {avg }}=\frac{1}{T} \int_{0}^{T} P(t) d t=\frac{1}{T R_{L}} \int_{0}^{T} V(t)^{2} d t
$$

The solution of the mathematical model is found using the numerical simulation. Specifically the current, $I$, passing through the coil is solved through the numerical simulation. The voltage, $V$, developed through the electrical circuit across the resistor is then computed. This voltage is used for the comparison of the experimental voltage output and to compute the instantaneous power harvestable through the electrical circuit.

\section{RESULTS AND DisCUSSIONS}

In this section, the results obtained from the SDOF TEG experiments are presented. The results and discussions here are focused on the frequency up-conversion and the harvestable power in the TEG.

The harvested energy can be maximized by placing the harvester coil centered at the region where the magnet undergoes high-frequency oscillations. Another way to maximize the power output is by both choosing a longer coil and positioning the coil in such a way that the harvester coil captures the whole oscillation of the oscillating magnet.

Two different linear springs were used in the SDOF twinkler. When the magnets detach and the magnet on the right side (refer to Figure 2 (a)) passes through the inductor coil, it generates current through the coil and this current is used to power a resistive load which results in the voltage output. Here the voltage output is presented and a fast Fourier transform (FFT) of the voltage is applied to find the output frequency of the SDOF TEG.

The total energy generated is then computed using the voltage output across the resistance load, from time $t=0$ till any given time $t$ within a period of time $T$ (period of the input $y(t)$ oscillation), by integrating the instantaneous power $P(t)$. For instance, the total energy generated in the harvester coil by the SDOF TEG from time $t=0$ till time $t=4.5$ seconds, correspondd to the point on the curve in Figure 8 at $t=4.5$ seconds. So, over one whole cycle of input oscillation the total energy harvested is the point on the curve in Figure 8 corresponding to time $t=10$ seconds.

The experimental voltage and energy output shown in Figures 7, and 8 respectively are in good agreement with the numerical results obtained from the analytical Models I and II. However, the FFT of the experimental voltage output in Figure 7 suggests that the model II is a slightly better analytical model than the model I. In particular, the twinkling frequency of the SDOF TEG from the experiment was $2.45 \mathrm{~Hz}$ where as the frequencies from the numerical models I and II are $3.2 \mathrm{~Hz}$ and $2.8 \mathrm{~Hz}$ respectively.

The peak instantaneous power is computed from the voltage output e.g. in a $2000 \Omega$ resistive load. Based on Figure 6, the SDOF TEG has a peak instantaneous power of $P_{\max } \approx(0.3 \mathrm{~V})^{2} / 2000 \Omega=0.045 \mathrm{~mW}$. The total cumulative energy produced in one cycle of low frequency input oscillation shown in Figure 8 is $0.045 \mathrm{mWs}$.

In the experimental setup, by swapping springs of different stiffnesses or using magnets of different magnetic strengths, this model can be extended to study various parameters. We have observed (not shown) that the stiffer the springs, the larger the amplitude of the low-frequency input needed to build up enough force to obtain the snap-through phenomenon of the twinkler. 


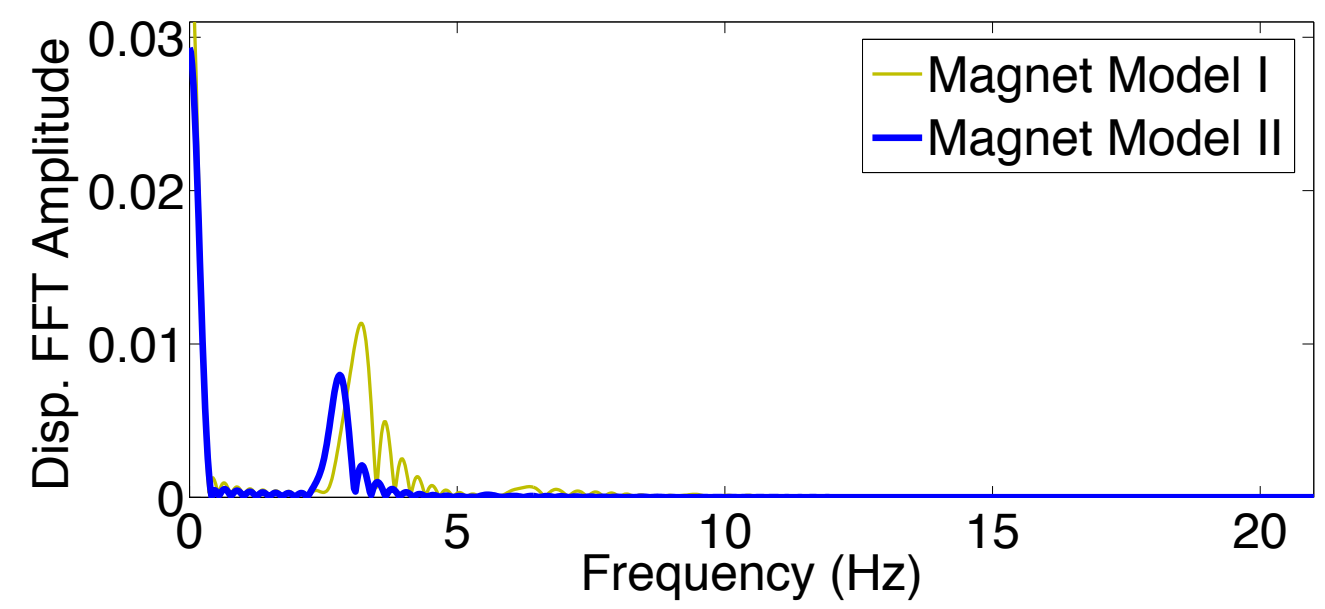

Fig. 5: The numerical frequency content in the displacement of the mass of the SDOF TEG over one period of input excitation of frequency $0.1 \mathrm{~Hz}$. The dominant output frequency $2.8 \mathrm{~Hz}$ shows the frequency up-conversion.

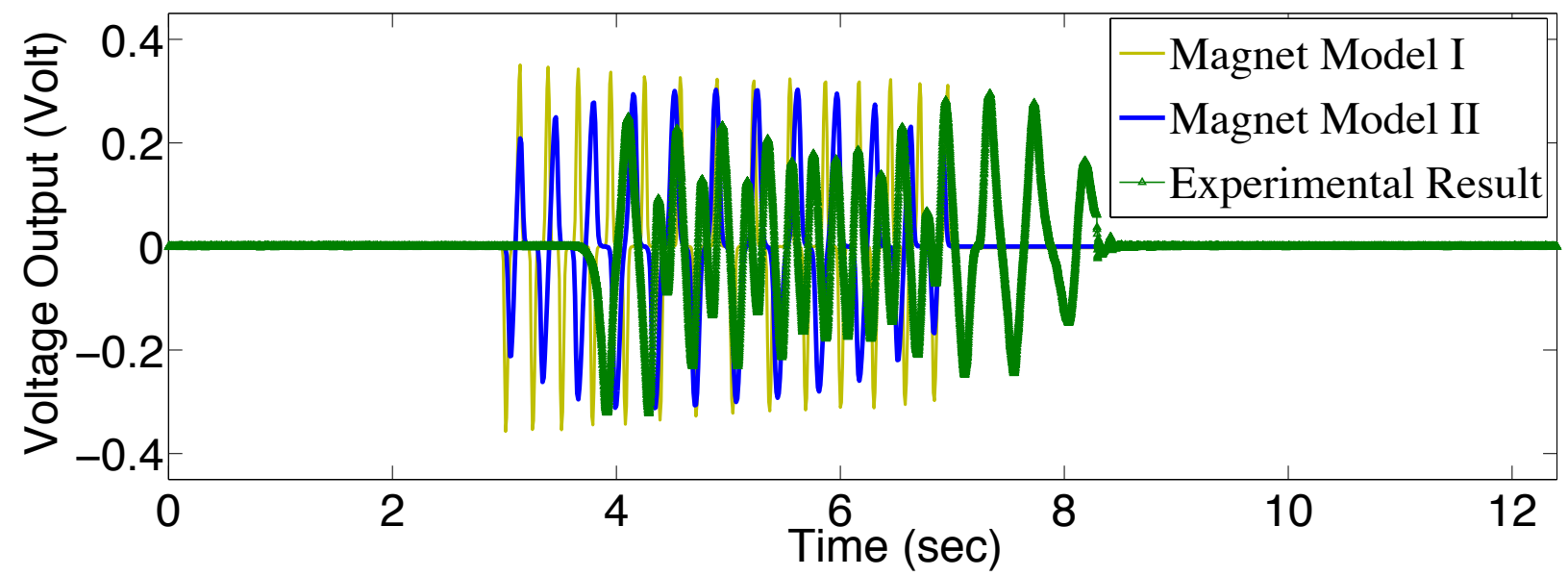

Fig. 6: The voltage computed from the numerical simulation for one cycle of $0.1 \mathrm{~Hz}$ input excitation at the end spring.

Both mathematical models can provide a reliable way to estimate power output and harvestable energy for given parameter values. By changing the electrical circuit parameters, one might be able to harvest more energy. For example, keeping all the parameters same and changing the electro-mechanical coupling parameter $\gamma$ from $1.1 \mathrm{As} / \mathrm{m}$ to $10 \mathrm{As} / \mathrm{m}$, total harvestable energy over one period of input oscillation (10 seconds) increases from $0.045 \mathrm{mWs}$ to $3.3 \mathrm{mWs}$. Using the best parameter values one would be able to harvest the optimum amount of energy from a low-frequency vibration source. A multiple-degree-of-freedom TEG would also enhance the amount of harvestable energy. Indeed we have performed 2-DOF experimental twinkling energy harvesting for various configurations, but the analytical and numerical formulations require significant development and are beyond scope of this paper.

There is some room for error in the experimental results as compared to the analytical and numerical results since the horizontal shaker can be affected by feedback from the end spring force which in turn creates a deviation to the input signal. This results in a not perfectly harmonic but a periodic input and feeds back to the circuit inducing a slight deviation to the expected output.

\section{CONCLUSIONS}

In this work, energy was harvested using coils and magnets by employing a snap-through nonlinearity which in turn generated the frequency up-conversion. The snap-through nonlinearity was designed using attracting magnets which could be attached and then released under the pull of a spring. The experiment demonstrated the up-conversion of a $0.1 \mathrm{~Hz}$ input oscillation into an output oscillation with a frequency of about $2.5 \mathrm{~Hz}$, which is 25 times the input frequency. Agreeable results were obtained by the numerical solution of the analytical models.

Two magnetic force models were derived and compared: one (Model I) based on point-dipole magnets, and another (Model II) accommodating the finite size of the magnets. Model II was slightly better than Model I at reproducing the experimental observations. However, both models present a reliable way to estimate the power output and harvestable energy for given parameters, and can be used for analytical and optimization studies. 


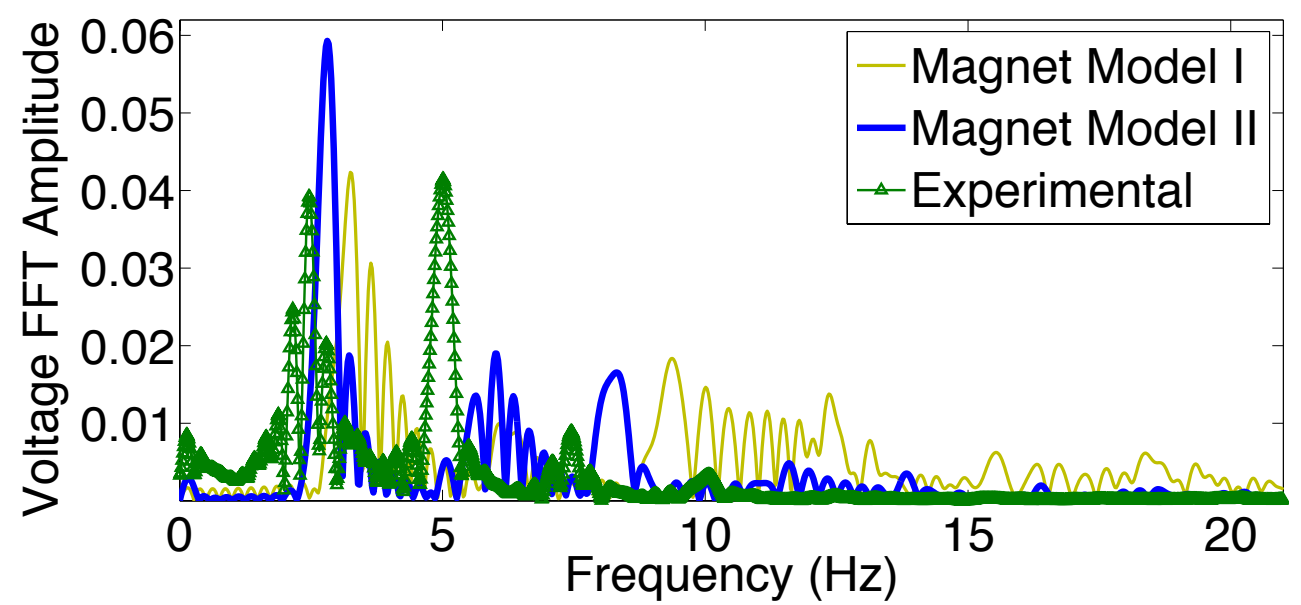

Fig. 7: The frequency content of the SDOF TEG from the experimental and numerically computed voltage output.

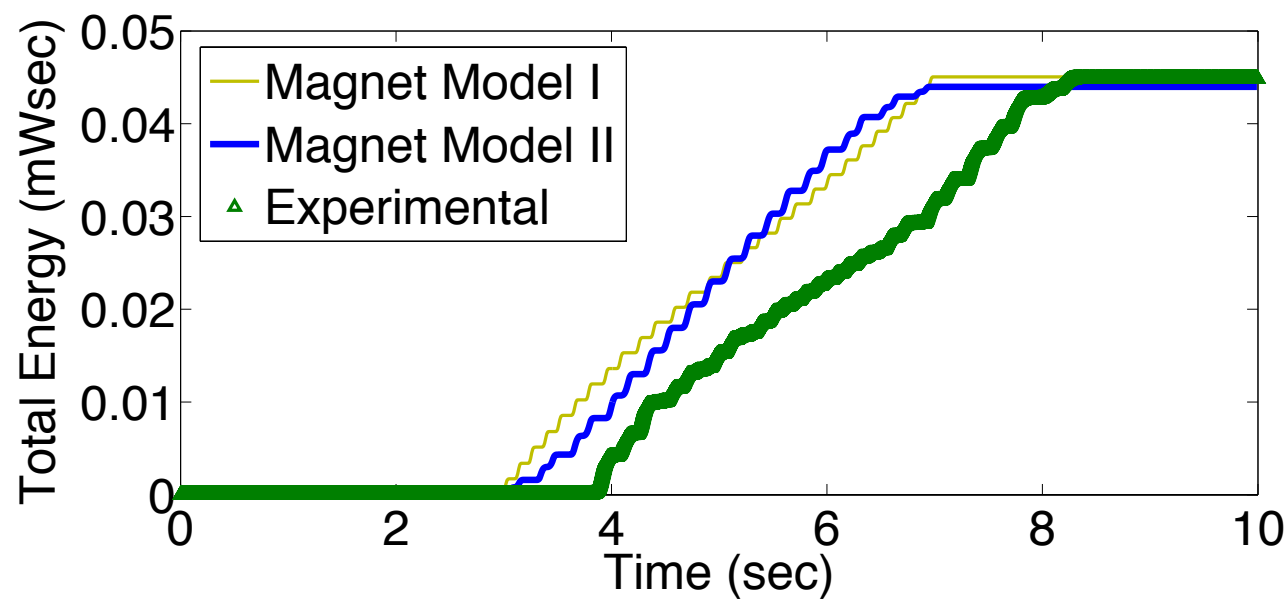

Fig. 8: A comparison between the experimentally harvested energy and the numerically computed energy for one cycle of 0.1 $\mathrm{Hz}$ input excitation.

Future work can examine the optimization of parameters, both in the mechanical set up and in the harvesting circuit, to maximize harvested energy. Furthermore, we have experimented with a two DOF TEG, and its mathematical model is a topic of ongoing work.

\section{ACKNOWLEDGMENTS}

This material is based upon work supported by the National Science Foundation under Grant No. CMMI-1030377. Any opinions, findings, and conclusions or recommendations expressed in this material are those of the authors and do not necessarily reflect the views of the National Science Foundation. We highly appreciate the help of Mr. Michael Mazzoleni during our experiment in collecting some of the parameter values used in this article.

\section{REFERENCES}

[1] Feeny, B. F., and Diaz, A. R., 2010. "Twinkling phenomena in snap-through oscillators". Journal of Vibration and Acoustics, 132 (061013)(5).

[2] Wang, Y. C., and Lakes, R. S., 2004. "Extreme stiffness systems due to negative stiffness elements". American Journal of Physics, 72, pp. 40-50.

[3] Puglisi, G., and Truskinovsky, L., 2000. "Mechanics of a discrete chain with bi-stable elements". Journal of the Mechanics and Physics of Solids, 48(1), pp. 1-27.

[4] Puglisi, G., and Truskinovsky, L., 2002. "Rate independent hysteresis in a bi-stable chain". Journal of the Mechanics and Physics of Solids, 50(2), pp. $165-187$.

[5] Puglisi, G., 2006. "Hysteresis in multi-stable lattices with non-local interactions". Journal of the Mechanics and Physics of Solids, 54(10), pp. 2060-2088.

[6] Sarafian, H., 2010. "Static electric-spring and nonlinear oscillations". Journal of Electromagnetic Analysis \& Applications (JEMAA), 2 (2), pp. 75-81.

[7] Sarafian, H., 2011. "Nonlinear oscillations of a magneto static spring-mass". Journal of Electromagnetic Analysis and Applications, 3, pp. 133-139.

[8] Stanton, S. C., Owens, B. A., and Mann, B. P., 2012. "Harmonic balance analysis of the bistable piezoelectric inertial generator". Journal of Sound and Vibration, 331(15), pp. 3617-3627.

[9] Roundy, S., and Wright, P. K., 2004. "A piezoelectric vibration based generator for wireless electronics". Smart Materials and Structures, 13, pp. 1131-1142.

[10] Sodano, H. A., Inman, D. J., and Park, G., 2005. "Comparison of piezoelectric energy harvesting devices for recharging batteries". Journal of Intelligent Material Systems and Structures, 16(10), pp. 799-807. 
[11] Liao, Y., and Sodano, H. A., 2008. "Model of a single mode energy harvester and properties for optimal power generation". Smart Materials and Structures, 17, p. 065026.

[12] Renno, J. M., Daqaq, M. F., and Inman, D. J., 2009. “On the optimal energy harvesting from a vibration source". Journal of Sound and Vibration, 320(1-2), pp. 386-405.

[13] Scruggs, J. T., and Behrens, S., 2011. "Optimal energy harvesting from low-frequency bistate force loadings". Journal of Vibration and Acoustics, 133, p. 011008 .

[14] Anton, S. R., and Sodano, H. A., 2007. "A review of power harvesting using piezoelectric materials (2003-2006)". Smart Materials and Structures, 16, R1.

[15] Stanton, S. C., Erturk, A., Mann, B. P., and Inman, D. J., 2010. "Nonlinear piezoelectricity in electroelastic energy harvesters: Modeling and experimental identification". Journal of Applied Physics, 108(7), pp. 074903-074903.

[16] Stanton, S. C., McGehee, C. C., and Mann, B. P., 2010. "Nonlinear dynamics for broadband energy harvesting: Investigation of a bistable piezoelectric inertial generator". Physica D: Nonlinear Phenomena, 239(10), pp. 640-653.

[17] Stanton, S. C., Erturk, A., Mann, B. P., Dowell, E. H., and Inman, D. J., 2012. "Nonlinear nonconservative behavior and modeling of piezoelectric energy harvesters including proof mass effects". Journal of Intelligent Material Systems and Structures, 23(2), pp. 183-199.

[18] Kim, S., Clark, W. W., and Wang, Q. M., 2005. "Piezoelectric energy harvesting with a clamped circular plate: experimental study". Journal of Intelligent Material Systems and Structures, 16(10), pp. 855-863.

[19] Mo, C., Radziemski, L. J., and Clark, W. W., 2010. "Experimental validation of energy harvesting performance for pressure-loaded piezoelectric circular diaphragms". Smart Materials and Structures, 19, pp. 75010-75016.

[20] Kerschen, G., McFarland, D. M., Kowtko, J. J., Lee, Y. S., Bergman, L. A., and Vakakis, A. F., 2007. "Experimental demonstration of transient resonance capture in a system of two coupled oscillators with essential stiffness nonlinearity". Journal of Sound and Vibration, 299(4-5), pp. 822-838.

[21] Quinn, D. D., Triplett, A. L., Bergman, L. A., and Vakakis, A. F., 2011. "Comparing linear and essentially nonlinear vibration-based energy harvesting". Journal of Vibration and Acoustics, 133(1), p. 011001.

[22] Quinn, D. D., Triplett, A. L., Vakakis, A. F., and Bergman, L. A., 2011. "Energy harvesting from impulsive loads using intentional essential nonlinearities". Journal of Vibration and Acoustics, 133, p. 011004.

[23] Mann, B. P., Barton, D. A., and Owens, B. A., 2012. "Uncertainty in performance for linear and nonlinear energy harvesting strategies". Journal of Intelligent Material Systems and Structures, 23(13), pp. 1451-1460.

[24] Panagopoulos, P. N., Vakakis, A. F., and Tsakirtzis, S., 2004. "Transient resonant interactions of finite linear chains with essentially nonlinear end attachments leading to passive energy pumping". International journal of solids and structures, 41(22), pp. 6505-6528.

[25] McFarland, D. M., Bergman, L. A., and Vakakis, A. F., 2005. "Experimental study of non-linear energy pumping occurring at a single fast frequency". International Journal of Non-Linear Mechanics, 40(6), pp. 891-899.

[26] Gourdon, E., Alexander, N. A., Taylor, C. A., Lamarque, C. H., and Pernot, S., 2007. "Nonlinear energy pumping under transient forcing with strongly nonlinear coupling: Theoretical and experimental results". Journal of Sound and Vibration, 300(3-5), pp. 522-551.

[27] Manevitch, L. I., Musienko, A. I., and Lamarque, C. H., 2007. "New analytical approach to energy pumping problem in strongly nonhomogeneous 2 DOF systems". Meccanica, 42(1), pp. 77-83.

[28] Panagopoulos, P. N., Gendelman, O., and Vakakis, A. F., 2007. "Robustness of nonlinear targeted energy transfer in coupled oscillators to changes of initial conditions". Nonlinear Dynamics, 47(4), pp. 377-387.

[29] Tsakirtzis, S., Panagopoulos, P. N., Kerschen, G., Gendelman, O., Vakakis, A. F., and Bergman, L. A., 2007. "Complex dynamics and targeted energy transfer in linear oscillators coupled to multi-degree-of-freedom essentially nonlinear attachments". Nonlinear Dynamics, 48(3), pp. $285-318$.

[30] Quinn, D. D., Gendelman, O., Kerschen, G., Sapsis, T. P., Bergman, L. A., and Vakakis, A. F., 2008. "Efficiency of targeted energy transfers in coupled nonlinear oscillators associated with 1:1 resonance captures: Part I". Journal of Sound and Vibration, 311(3-5), pp. 1228-1248.

[31] Sapsis, T. P., Vakakis, A. F., Gendelman, O. V., Bergman, L. A., Kerschen, G., and Quinn, D. D., 2009. "Efficiency of targeted energy transfers in coupled nonlinear oscillators associated with 1:1 resonance captures: Part II, analytical study". Journal of Sound and Vibration, 325(1-2), pp. 297-320.

[32] Vakakis, A., Gendelman, O., Bergman, L., McFarland, D., Kerschen, G., and Lee, Y. S., 2009. Nonlinear Targeted Energy Transfer in Mechanical and Structural Systems: I and II. Springer, Berlin.

[33] Bellet, R., Cochelin, B., Herzog, P., and Mattei, P. O., 2010. "Experimental study of targeted energy transfer from an acoustic system to a nonlinear membrane absorber". Journal of Sound and Vibration, 329(14), pp. 2768-2791.

[34] Owens, B. A., and Mann, B. P., 2012. "Linear and nonlinear electromagnetic coupling models in vibration-based energy harvesting". Journal of Sound and Vibration, 331(4), pp. 922-937.

[35] Priya, S., and Inman, D. J., 2008. Energy harvesting technologies. Springer, New York.

[36] Stephen, N. G., 2006. "On energy harvesting from ambient vibration". Journal of Sound and Vibration, 293(1-2), pp. 409-425.

[37] Mann, B. P., and Sims, N. D., 2009. "Energy harvesting from the nonlinear oscillations of magnetic levitation". Journal of Sound and Vibration, 319(1-2), pp. 515-530.

[38] Zheng, R., Nakano, K., Hu, H., Su, D., and Cartmell, M. P., 2014. "An application of stochastic resonance for energy harvesting in a bistable vibrating system". Journal of Sound and Vibration, 333(12), pp. 2568-2587.

[39] Mann, B. P., and Owens, B. A., 2010. "Energy harvesting from the nonlinear oscillations of magnetic levitation". Journal of Sound and Vibration, 329(9), pp. 1215-1226.

[40] Harne, R. L., and Wang, K. W., 2014. "On the fundamental and superharmonic effects in bistable energy harvesting". Journal of Intelligent Material Systems and Structures, 25, pp. 937-950.

[41] Harne, R. L., and Wang, K. W., 2013. "A review of the recent research on vibration energy harvesting via bistable systems". Smart Materials and Structures, 22(2), p. 023001.

[42] Harne, R. L., Thota, M., and Wang, K. W., 2013. "Bistable energy harvesting enhancement with an auxiliary linear oscillator". Smart Materials and Structures, 22(12), p. 125028.

[43] Erturk, A., and Inman, D. J., 2011. "Broadband piezoelectric power generation on high-energy orbits of the bistable Duffing oscillator with electromechanical coupling". Journal of Sound and Vibration, 330, pp. 2339-2353.

[44] Masana, R., and Daqaq, M. F., 2012. "Energy harvesting in the super-harmonic frequency region of a twin-well oscillator". Journal of Applied Physics, 111(4), p. 044501.

[45] Masana, R., and Daqaq, M. F., 2011. "Relative performance of a vibratory energy harvester in mono-and bi-stable potentials". Journal of Sound and Vibration, 330(24), p. 044501

[46] Galchev, T., Kim, H., and Najafi, K., 2011. "Micro power generator for harvesting low-frequency and nonperiodic vibrations". Journal of Microelectromechanical Systems, 20(4), pp. 852-866.

[47] Yerkes, R. M., 1905. "The sense of hearing in frogs". Journal of Comparative Neurology and Psychology, 15(4), pp. 279-304.

[48] Hudspeth, A. J., and Lewis, R. S., 1988. "A model for electrical resonance and frequency tuning in saccular hair cells of the bull-frog, rana catesbeiana". The Journal of physiology, 400(1), pp. 275-297.

[49] Ahn, K. H., 2009. "A bio-inspired electromechanical system: Artificial hair cell". Electron Transport in Nanosystems, pp. 351-359.

[50] Lee, C., and Park, S., 2012. "A mechanical model of stereocilia that demonstrates a shift in the high-sensitivity region due to the interplay of a negative stiffness and an adaptation mechanism”. Bioinspiration \& Biomimetics, 7(4), p. 046013. 
[51] Panigrahi, S. R., Feeny, B. F., and Diaz, A. R., 2014. "Degenerate "star" bifurcations in a twinkling oscillator". Journal of Vibration and Acoustics, 136(2), p. 021004.

[52] Panigrahi, S. R., Feeny, B. F., and Diaz, A. R., 2014. “'Eclipse' bifurcation in a twinkling oscillator". Journal of Vibration and Acoustics, 136(3), p. 034504

[53] Gilbert, W., 1958. De Magnete (1600), Translated by P. Fleury Mottelay. Courier Corporation.

[54] Vokoun, D., Beleggia, M., Heller, L., and Sittner, P., 2009. "Magnetostatic interactions and forces between cylindrical permanent magnets". Journal of Magnetism and Magnetic Materials, 321(22), pp. 3758-3763.

[55] Beleggia, M., Tandon, S., Zhu, Y., and De Graef, M., 2004. "On the magnetostatic interactions between nanoparticles of arbitrary shape". Journal of Magnetism and Magnetic Materials, 278(1), pp. 270-284.

[56] Vokoun, D., Beleggia, M., Rahman, T., Hou, H. C., and Lai, C. H., 2008. "The two-spin model with dipolar interactions for the exchange coupled composite media". Journal of Applied Physics, 103(7), p. 07f520.

[57] Abramowitz, M., and Stegun, I. A., 1970. Handbook of mathematical functions. Dover, New York. 

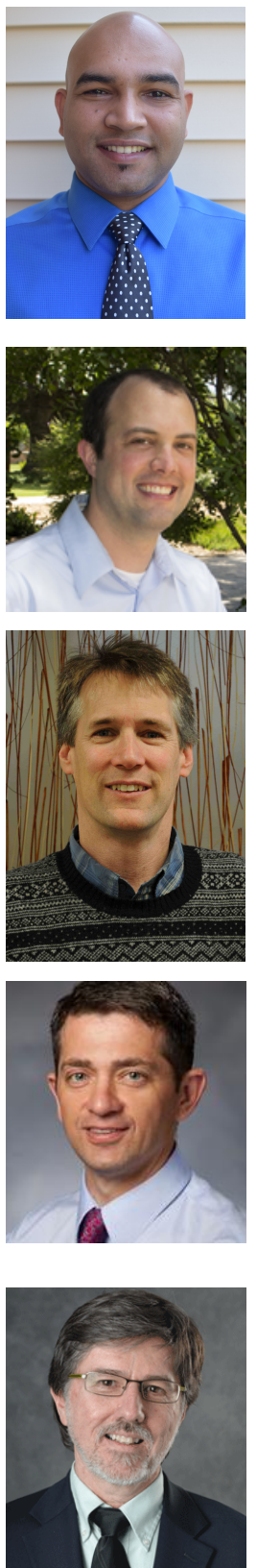

Dr. Smruti R. Panigrahi is currently working as a Controls Research Engineer and Data Scientist in Research and Advanced Engineering at Ford Motor Company. He earned his Ph.D. in Mechanical Engineering and M.S. in Applied Mathematics from Michigan State University in 2014 and 2013 respectively. He holds a masters' degree in Mechanical Engineering specializing in Virtual Reality from Washington State University and B.S. in Manufacturing Science and Engineering from the Indian Institute of Technology, Kharagpur, India. His research interests are in vibration, dynamics and control, energy harvesting, metamaterials, artificial intelligence, autonomous vehicles, augmented reality, deep/machine learning, and intelligent transportation systems.

Prof. Brian P. Bernard is currently serving as an Assistant Professor in the Department of Mechanical Engineering at Schreiner University. He earned his Ph.D. in Mechanical Engineering from Duke University in 2014. His research interests are in wave propagation, design of bandage structures, metamaterials, energy harvesting, bifurcation and stability analysis, and experimental study of energy and wave propagation. biography text here.

Prof. Brian F. Feeny earned his Ph.D. in Theoretical and Applied Mechanics from Cornell University in 1990. He then did postdoctoral research at ETH Zurich, Switzerland. He has been a faculty member at Michigan State University since 1992. He is a Fellow of the American Society of Mechanical Engineers (ASME). He has served as the Chair of the ASME Technical Committee on Vibration and Sound and as an associate editor of the ASME Journal of Vibration and Acoustics and Journal of Computational and Nonlinear Dynamics. His research interests are in nonlinear dynamics and vibration, chaos, wave propagation, system identification, proper orthogonal decomposition, friction dynamics, wind turbines and energy harvesting.

Prof. Brian P. Mann is an ASME fellow and a Professor in the department of Mechanical Engineering and Materials Science at Duke University. He received his D.Sc. degree from Washington University in 2003. He is a member and fellow of American Society of Mechanical Engineers. His expertise and research interests are in experimental energy harvesting, nonlinear dynamics and vibration, numerical and analytical method development, stability analysis, and chaos.

Prof. Alejandro R. Diaz earned his B.S.E., M.S., and Ph.D. in Aerospace Engineering from University of Michigan in 1978, 1979, and 1982 respectively. Since then he has been serving as a professor at Michigan State University. He is a member and fellow of American Society of Mechanical Engineers. He also served as the head of the Mechanical Engineering Department at MSU from 2010 to 2015. His research interests are in topology optimization, optimal design of structures and materials, design of bandgap structures, origami inspired frequency selective surfaces, finite element methods, metamaterials, and energy scavenging. 